Commentary

\title{
Linear and cooperative signaling: roles for Stat proteins in the regulation of cell survival and apoptosis in the mammary epithelium Bernd Groner and Lothar Hennighausen*
}

\author{
Georg Speyer Haus, Institute for Biomedical Research, Frankfurt am Main, Germany \\ and ${ }^{*}$ National Institutes of Health, Bethesda, Maryland, USA
}

\begin{abstract}
The mammary epithelium undergoes cyclical periods of cellular proliferation, differentiation and regression. These processes are under the control of the hormones secreted during pregnancy, lactation and involution. Signaling pathways have been identified that connect the hormonal stimuli with the transcription of genes responsible for the determination of the cellular fate. The kinetics of induction and deinduction have suggested that cytokineactivated Stat proteins play a crucial role. Stat5 is strongly activated towards the end of pregnancy, persists in an activated state during pregnancy and is rapidly inactivated after cessation of suckling. Stat3 activation is hardly detectable during lactation, but is strongly induced at the onset of involution. The phenotypes of mice in which these genes have been inactivated through homologous recombination corroborate some of the functional assignments deducted from the activation pattern. Stat3 activation seems to be a driving force in the induction of apoptosis early in the involution period.
\end{abstract}

Keywords: apoptosis, mammary gland, Stat3, tissue specific knock-out

\section{Signaling pathways and new opportunities for drug discovery}

Nearly all cellular decisions are determined by extracellular signals (eg hormones, growth factors, surface proteins of neighboring cells or extracellular matrix components). These signals are thought to be converted into enzymatic activities and patterns of gene transcription that determine the functions and phenotypes of cells. Signal transduction is controlled by intracellular signaling cascades and the regulation of latent transcription factors. The identification of crucial components, such as specific receptors for signals at the cell surface and linked signal transduction molecules, has led to a basic understanding of, for example, the function of growth factors such as epidermal growth factor or platelet-derived growth factor, hematopoi- etic cytokines, hormones recognizing receptors coupled to $\mathrm{G}$ proteins and steroid hormone action.

Although we are still far from a comprehensive picture especially, the identification of postulated, decisive target genes specifying particular cellular parameters is not sufficiently advanced - the concept has proven valuable. Linear chains of events have been described that link defined extracellular signals to the transcription of individual genes. These insights have nourished the confidence that a network of interactions can be established which will allow very specific modes of interference and the development of new anticancer drugs. These drugs should preferentially target the growth propelling signals in a population of tumor cells with particular genetic abberations, or alterna-

IL-6 = interleukin-6. 
(a)

Cell Death and Remodeling of Mammary tissue

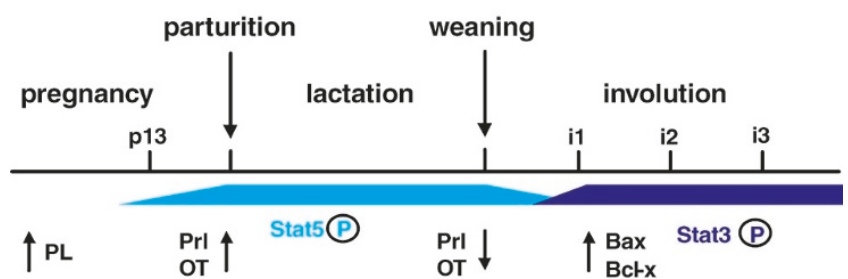

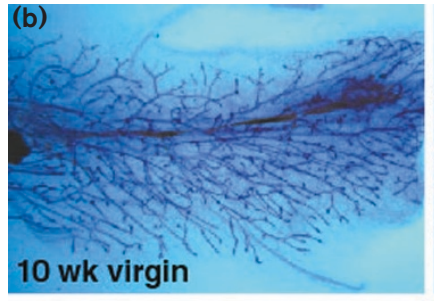
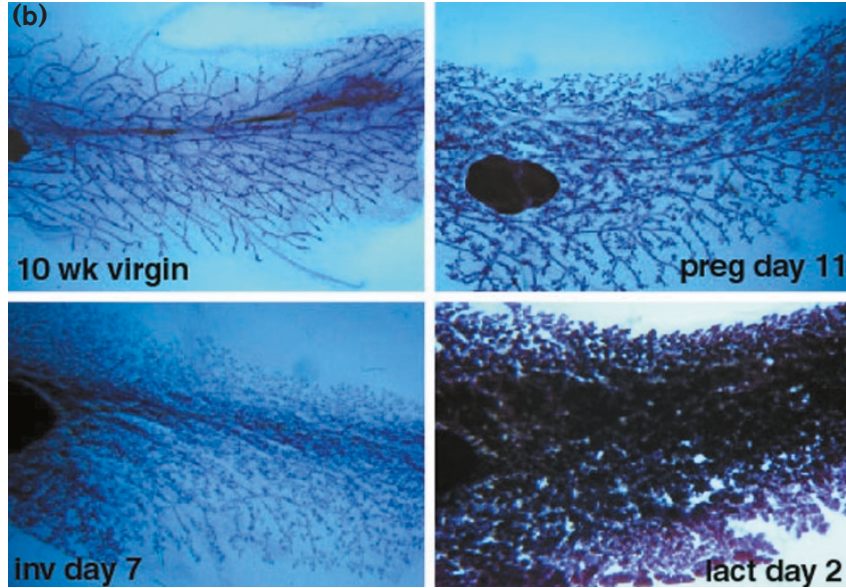

Stages of mammary gland development. (a) The development of the mouse mammary gland can be divided into five stages (pregnancy, parturition, lactation, weaning, involution) and crucial hormonal components required for each transition have been identified [22]. Placental lactogen (PL) concentrations increase during pregnancy, and prolactin (Prl) and oxytocin (OT) concentrations rise during lactation, sharply declining after weaning. P13, pregnancy day 13 ; i1, involution day 1 ; i2, involution day 2 ; i3, involution day 3 . (b) Histological whole mount analysis of mouse mammary gland from virgin mice, pregnant, lactating and involuting mice. Mammary tissues from virgin mice (upper left panel), mice at day 11 of pregnancy (upper right panel), day 2 of lactation (lower right panel) and day 7 of involution (lower left panel) were fixed and stained with Carmine Red. Extensive cellular proliferation occurs during pregnancy; quiescent, terminally differentiated secretory alveolar cells are responsive to the lactogenic hormones and responsible for milk production during lactation; massive cell death characterizes the involution phase.

tively activate cellular suicide programs. It is hoped that the principle exemplified by tamoxifen can be emulated. A growth limiting step, in this case the activation of the estrogen receptor, can be identified and exploited for targeted interference. In the most favorable cases, this step is rather restricted to the regulation of the growth of tumor cells, thus its inhibition will cause few undesirable side effects.

\section{Stat 3 and the regulation of apoptosis in hematopoietic and mammary epithelial cells}

A prerequisite for such an approach is the detailed understanding of the function of individual signaling pathways in normal cells and the consequences of their deregulation in specific cell types. Especially, signals regulating such basic cellular properties as survival, proliferation, differentiation and apoptosis have caught the fancy of molecular oncologists. If individual signaling components prove to be rate limiting for such complex genetic programs, they would constitute outstanding drug targets. Important progress has also been made in the development of the tools that allow the evaluation of such molecules. Inactivation of individual genes in embryonic stem cells by homologous recombination and observation of the consequences of such a mutagenic event in animals is the method of choice.

Chapman et al have applied this technology to the investigation of a member of the Stat family of transcription factors (Stat3) in the context of the mammary gland [1]. Stat factors are latent transcription factors and comprise a family of seven genes encoding proteins of similar domain structures and modes of activation. They are activated by receptor-associated cytoplasmic tyrosine kinases (Jak) through phosphorylation on a single tyrosine residue. Phosphorylated Stat molecules can form dimers, translocate to the nucleus, bind to specific response elements in the promoter of target genes and alter the rate of transcription $[2,3]$.

Stat3 was originally discovered as the acute phase response factor, a transcription factor activated by IL-6 and its receptor in liver cells [4]. Subsequently, many signals have been shown to relay their effects through Stat3. The hint that Stat3 might also regulate growth properties of cells came from studies which showed that cellular transformation with transfected oncogenes or infection with tumor viruses were often accompanied by the constitutive activation of Stat3, along with Stat5 and, sometimes, Stat1. The use of dominant negative variants of Stat3 showed that at least NIH 3T3 cell transformation by v-src is dependent on the function of Stat3 [5]. A Stat3 variant with two amino acid substitutions functions as an oncogene in tissue culture cells and nude mice [6]. Constitutive activation of Stat3 was also found in primary human leukemia cells, further implicating Stat3 in the process of malignant transformation [7].

These observations were complemented by investigations into the connections between Stat3 and apoptosis regula- 
tors. A complex network of cytokines, receptors, cytoplasmic signaling molecules and transcription factors control this process, and members of the Bcl-2 family of proteins play central roles in the regulation of apoptosis [8]. A balance of pro- and anti-apoptotic influences determines the outcome. Disturbances in this balance may confer resistance to physiological signals of apoptosis and lead to cellular transformation. The induction of Stat3 by IL-6 in myeloma cells has been shown to protect these cells from apoptotic stimuli emanating from the Fas (CD95/Apo-1) receptor or chemotherapeutic agents [9]. In this cell type, Stat3 activation results in the transcriptional induction of the $b c /-x$ gene promoter and high levels of the anti-apoptotic protein Bcl-xL. Thus, elevated levels of Stat3 appear to contribute directly to malignancy in multiple myeloma cells by the prevention of apoptosis $[10,11]$.

The mammary gland is a favorable organ to study molecular mechanisms governing apoptosis (Fig. 1a). Pregnancy induces massive proliferation of mammary epithelial cells and the development of alveolar structures. The differentiated secretory epithelium produces large amounts of milk during the suckling and lactation period [12]. Gene deletion studies in mice have demonstrated that Stat5a is required for cell proliferation, differentiation and survival during pregnancy and lactation. After cessation of suckling, Stat5 is rapidly inactivated and the lobulo-alveolar structures collapse and reductive remodeling of the gland sets in (Fig. 1b). This involution period is characterized by proteolytic degradation of the extracellular matrix and elimination of the majority of the secretory epithelial cells by apoptosis $[13,14]$. If Stat3 has a role in this process and acts in a similarly anti-apoptotic fashion as myeloma cells, one might expect it to be activated during the lactation period and downregulated during involution. The observation that activated Stat3 is not detectable in the lactating gland but induced shortly after cessation of suckling (Fig. 2) suggests otherwise. Advanced knockout technology showed that Stat3 actually fulfills the opposite function in these cells, ie it promotes apoptosis in the postsuckling state.

Chapman et al arrived at this conclusion through observation of a mouse strain in which the Cre-lox recombination system has been directed towards the elimination of Stat3 function, specifically in mammary epithelial cells. For this purpose, the Cre recombinase was expressed under the control of the beta-lactoglobulin gene promoter, a promoter that is fully activated in differentiated mammary epithelial cells under the control of lactogenic hormones [15]. The Stat3 gene was present in a heterozygous state. One allele was inactivated through standard homologous recombination (a null allele) [16], the second allele was floxed, ie loxP sites were introduced around the tyrosine phosphorylation domain [17]. These loxP sites recombine in the presence of Cre recombinase, which results in the deletion of the tyro-
Figure 2

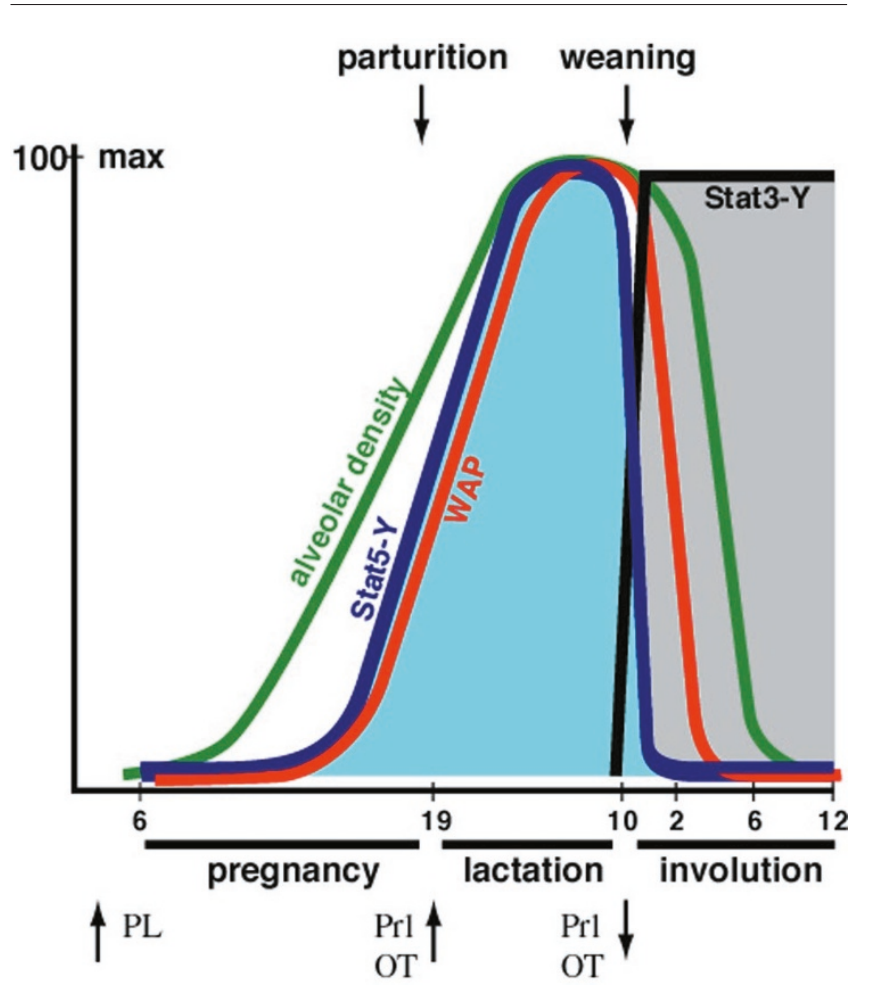

Hormonal signals and the activation of transcription factors mediating mammary gland development. During the pregnancy stage, alveolar density continuously increases. Towards the end of pregnancy, starting at day 13, Stat $5 \mathrm{a} / \mathrm{b}$ are induced by tyrosine phosphorylation and can be detected in their DNA binding configuration. Activated Stat5 persists during lactation, but rapidly declines after cessation of suckling [23]. Activated Stat3 is not detectable during the lactation phase, but is rapidly induced to maximal levels several hours into the weaning period [24]. Within the same time, the levels of $b c l-x$ and bax increase [25]. Milk protein synthesis (WAP) and alveolar density decrease during involution.

sine phosphorylation domain and the inactivation of the second allele. Chapman et al show that this process occurs efficiently in differentiated lobular alveolar cells, initially without phenotype. Upon cessation of suckling, however, they observe that the wild-type cells, capable of Stat3 induction, show a much higher level of apoptosis and a more rapid onset of involution. Protection from apoptosis in Stat3-deficient epithelial cells is transient and involution sets in with a delay of about 3 days. Eventually, it catches up the involution process observed in mice with an intact Stat3 pathway. Unlike in myeloma cells, where Stat3 activates the $b c l-x$ gene, encoding a survival protein and protecting against apoptosis, the $b c l-x$ gene does not appear to be a target gene in mammary epithelial cells.

\section{Cooperation of signaling pathways}

Examination of additional molecular parameters that might explain this delay in apoptosis induction in Stat3-deficient 
mammary epithelium turned up two interesting differences between the Stat3 expressing and nonexpressing glands. The activated Stat5 molecule is usually rapidly inactivated upon cessation of suckling, a process triggered by the lack of the suckling stimulus and a decrease in the circulating level of prolactin [18]. The forced expression of an activated Stat5 variant in the mammary cells seems protective against apoptosis at this stage. The observation in the paper that Stat5 persists longer in the Stat3 nonexpressing cells than in the wild-type cells may hint at an underlying mechanism for apoptosis protection. A connection between the regulation of individual members of the Stat family, ie a hierarchy of action, downregulation of Stat5 in the presence of Stat3, could be envisaged. Alternatively, Stat3 could generate a growth promoting signal that results in the induction of apoptosis in the absence of a complementary survival signal. Analogous observations have been made in oncogene expressing cells in the absence of serum. This process could accelerate the death of mammary epithelial cells in Stat3 expressing mice and slow it down, but not prevent it, in Stat3-deficient mice. The second interesting possibility discussed involves the levels of IGFBP-5. This protein might be induced by Stat3, sequesters IGF-I and prevents the activation of the IGF-I receptor. The activated receptor exerts a strong anti-apoptotic action $[19,20]$.

The conclusion that Stat3 can exert an anti-apoptotic effect in myeloma cells and a pro-apoptotic effect in secretory mammary epithelial cells seems initially contradictory. Although dealing with different cell types, it seems unlikely that they should have developed opposing mechanisms of apoptosis regulation. Differential effects of a single signal on distinct cell types, however, are not unknown. Glucocorticoid hormones, for example, are potent inducers of apoptosis in lymphocytes, but do not kill fibroblasts or epithelial cells. Even slight proliferative responses to the hormone have been observed in these cell types. The c-myc gene can contribute to proliferation and transformation in cells grown under serum conditions, but can induce apoptosis if a serum derived signal is missing [21]. High levels of activated Stat3 are not only observed during involution, but also during puberty, a time of rapid ductal growth and branching. This suggests that Stat3 may serve additional functions during puberty, which will be uncovered upon deletion of the Stat3 gene in mammary tissue at earlier developmental stages. The use of a Cre gene under control of the mouse mammary tumor virus long terminal repeat, which is already active in early ductal structures, should provide further insight.

The key to the understanding of differential and cellspecific effects might be found in the integration of signals and activated pathways that each cell type encounters at any time. Lateral signaling, ie the activation of receptors through unconventional ligands, and the intracellular inter- action and cooperation of signaling components will complement our picture of linear pathways in the future. In addition, duration and intensity of signals have to be taken into account. The diversity of signaling-induced phenotypes through cooperation of signaling pathways and components is still poorly appreciated. These parameters will provide explanations for cell type specific effects of individual signaling components and a code of signal interactions might ensue. This might become comparable with other codes used in cells to generate diversity through combinatorial mechanisms from a limited set of components. Its elucidation will certainly be labor intensive, but potentially rewarding since it increases the possibilities for targeted interference and the development of useful drugs.

\section{Acknowledgement}

The authors would like to thank Gertraud Robinson for the whole mount images shown in Figure 1.

\section{References}

1. Chapman RS, Lourenco PC, Tonner E, et al: Suppression of epithelial apoptosis and delayed mammary gland involution in mice with a conditional knockout of Stat3. Genes Dev 1999, 13:2604-2616.

2. Ihle JN: Stats: signal transducers and activators of transcription. Cell 1996, 84:331-334.

3. Darnell JE: Stats and gene regulation. Science 1997, 277:16301635.

4. Akira S, Nishio $Y$, Inoue M, et al: Molecular cloning of APRF, a novel IFN-stimulated gene factor 3 p91-related transcription factor involved in the gp130-mediated signaling pathway. Cell 1994, 77: 63-71.

5. Bromberg JF, Horvath CM, Besser D, Lathem WW, Darnell JE: Stat3 activation is required for cellular transformation by v-src. $\mathrm{Mol} \mathrm{Ce} / \mathrm{l}$ Biol 1998, 18:2553-2558.

6. Bromberg JF, Wrzeszczynska MH, Devgan G, et al: Stat3 as an oncogene. Cell 1999, 98:295-303.

7. Gouilleux-Gruart V, Gouilleux F, Desaint C, et al: Stat-related transcription factors are constitutively activated in peripheral blood cells from acute leukemia patients. Blood 1996, 87:1692-1697.

8. Adams JM, Cory S: The Bcl-2 protein family: arbiters of cell survival. Science 1998, 281:1322-1326.

9. Fukada T, Hibi M, Yamanaka Y, et al: Two signals are necessary for cell proliferation induced by a cytokine receptor gp130: involvement of Stat3 in anti-apoptosis. Immunity 1996, 5:449-460.

10. Catlett-Falcone R, Landowski TH, Oshiro MM, et al: Constitutive activation of Stat3 signaling confers resistance to apoptosis in human U266 myeloma cells. Immunity 1999, 10:105-115.

11. Fukada $T$, Ohtani $T$, Yoshida $Y$, et al: Stat3 orchestrates contradictory signals in cytokine-induced $\mathrm{G} 1$ to $\mathrm{S}$ cell-cycle transition. EMBO J 1998, 17:6670-6677.

12. Medina $D$ : The mammary gland: a unique organ for the study of development and tumorigenesis. J Mammary Gland Biol Neoplasia 1996, 1:5-19.

13. Chepko G, Smith GH: Three division-competent, structurally distinct cell populations contribute to murine mammary epithelial renewal. Tissue Cell 1997, 29:239-253.

14. Li ML, Liu XW, Robinson GW, et al: Mammary-derived signals activate programmed cell death during the first stage of mammary gland involution. Proc Natl Acad Sci USA 1997, 94:3425-3430.

15. Selbert S, Bentley DJ, Melton DW, et al: Efficient BLG-Cre mediated gene deletion in the mammary gland. Transgenic Res 1998, 7: 387-396.

16. Takeda K, Noguchi K, Shi W, et al: Targeted disruption of the mouse Stat3 gene leads to early embryonic lethality. Proc Natl Acad Sci USA 1997, 94:3801-3804.

17. Takeda K, Kaisho T, Yoshida N, Takeda J, Kishimoto T, Akira S: Stat3 activation is responsible for IL-6-dependent $T$ cell proliferation through preventing apoptosis: generation and characterization of $\mathrm{T}$ cell-specific Stat3-deficient mice. J Immunol 1998, 161:4652-4660. 
18. Quarrie LH, Addey CP, Wilde CJ: Programmed cell death during mammary tissue involution induced by weaning, litter removal, and milk stasis. J Cell Physiol 1996, 168:559-569.

19. Neuenschwander S, Schwart A, Wood TL, Roberts Jr CT, Hennighausen L, Le Roith D: Involution of the lactating mammary gland is inhibited by the IGF system in a transgenic mouse model. $J$ Clin Invest 1996, 97:2225-2232.

20. Tonner E, Barber MC, Travers MT, Logan A, Flint DJ: Hormonal control of insulin-like growth factor binding protein $\mathbf{5}$ production in the involuting mammary gland of the rat. Endocrinology 1997, 138:5101-5107.

21. Evan Gl, Littlewood TD: The role of c-myc in cell growth. Curr Opin Genet Dev 1993, 3:44-49.

22. Topper YJ, Freeman CS: Multiple hormone interactions in the developmental biology of the mammary gland. Physiol Rev 1980, 60:1049-1056.

23. Liu XW, Robinson GW, Wagner KU, Garrett L, Wynshaw B, Hennighausen $L$ : Stat5a is mandatory for adult mammary gland development and lactogenesis. Genes Dev 1997, 11:179-186.

24. Philp JC, Burdon TG, Watson CJ: Differential activation of Stats 3 and 5 during mammary gland development. FEBS Lett 1996, 396: 77-80.

25. Heermeier K, Benedict M, Li ML, Furth $P$, Nunez G, Hennighausen L: $\mathrm{Bax}$ and $\mathrm{Bcl}-\mathrm{x}_{\mathrm{s}}$ are induced at the onset of apoptosis in involuting mammary epithelial cells. Mech Dev 1996, 56:197-207.

Author's affiliation: Bernd Groner (Georg Speyer Haus, Institute for Biomedical Research, Frankfurt am Main, Germany) and Lothar Hennighausen (Laboratory of Genetics and Physiology, National Institutes of Health, Bethesda, Maryland, USA)

Correspondence: Bernd Groner, Georg Speyer Haus, Institute for Biomedical Research, Paul Ehrlich Strasse 42-44, D-60596 Frankfurt am Main, Germany. Tel: +49 696339 5180; fax: +4969 6339 5185; e-mail: groner@em.uni-frankfurt.de 